\title{
Estudo comparativo da função e qualidade de vida de pacientes submetidos à artroplastia total do joelho com plataformas tibiais fixa e móvel
}

\section{Comparative Study of the Function and Quality of Life of Patients Submitted to Total Knee Arthroplasty with Fixed and Mobile Tibial Platforms}

\author{
Daiane Cavenaghi Nacca ${ }^{1}$ Joicemar Tarouco Amaro ${ }^{1}$ Mateus Kenji Christo Miyahira2 ${ }^{2}$ \\ João Victor Novaretti ${ }^{10}$ Diego Costa Astur ${ }^{1 \odot}$ Moisés Cohen ${ }^{1}$ \\ 1 Departamento de Ortopedia e Traumatologia, Escola Paulista de \\ Medicina, Universidade Federal de São Paulo, São Paulo, São Paulo, Brasil \\ 2 Instituto Cohen de Ortopedia e Reabilitação e Medicina do Esporte, \\ São Paulo, São Paulo, Brasil \\ Endereço para correspondência Mateus Kenji Christo Miyahira, \\ Instituto Cohen, Av Lineu de Paula Machado 660, Cidade Jardim, \\ São Paulo, SP, 05601-000, Brasil \\ (e-mail: kenjimiyahira23@hotmail.com).
}

Rev Bras Ortop 2021;56(1):53-60.

\section{Resumo}

\section{Palavras-chave}

- artroplastia do joelho

- qualidade de vida

- osteoartrose

Objetivo Comparar a função e qualidade de vida dos pacientes submetidos a artroplastia total de joelho (ATJ) com plataforma tibial fixa e plataforma tibial móvel. Métodos Foram avaliados 240 pacientes com diagnóstico de osteoartrose de joelho, em um ensaio clínico, randomizados em dois grupos: grupo A, composto por 120 pacientes submetidos a AT] com plataforma tibial fixa, e grupo B, formado por 120 pacientes com plataforma móvel. Todos foram avaliados de acordo com a função e qualidade de vida pelos questionários de Western Ontario and McMaster Universities Arthritis Index (WOMAC) e Short Form Health Survey(SF-36), e escores de dor, por meio da escala visual analógica (EVA) de dor, no pré-operatório e com 6meses, 1ano, 2anos, 4anos e 8anos de cirurgia. Resultados Com relação aos diversos domínios do SF-36, o comportamento médio dos escores de capacidade funcional, aspectos físicos, dor e aspectos emocionais foram estatisticamente diferentes ao longo do seguimento, em ambos os grupos. Os demais domínios de qualidade de vida não apresentaram diferenças. Assim como na EVA de dor, o escore médio do WOMAC de dor apresentou melhora ao longo do seguimento em ambos os grupos. Entretanto, com dois anos de seguimento, foram estaticamente piores no grupo A, se igualando ao grupo B nos outros momentos de acompanhamento.

Conclusão Com 2anos de pós-operatório, os escores de dor do WOMAC e daEVA foram piores no grupo submetido aAT] com plataforma tibial fixa. Porém, as diferenças não permaneceram no médioprazo, sugerindo que a artroplastia com plataforma tibial móvel tem uma vantagem no curto prazo, podendo auxiliar no processo de reabilitação.

recebido

08 de Julho de 2019

aceito

27 de Janeiro de 2020

Publicado online

Julho 8,2020
DOI https://doi.org/

10.1055/s-0040-1709989. ISSN 0102-3616. (c) 2020. Sociedade Brasileira de Ortopedia e Traumatologia. All rights reserved.

This is an open access article published by Thieme under the terms of the Creative Commons Attribution-NonDerivative-NonCommercial-License, permitting copying and reproduction so long as the original work is given appropriate credit. Contents may not be used for commercial purposes, or adapted, remixed, transformed or built upon. (https://creativecommons.org/ licenses/by-nc-nd/4.0/)

Thieme Revinter Publicações Ltda., Rua do Matoso 170, Rio de Janeiro, RJ, CEP 20270-135, Brazil 


\begin{abstract}

\section{Keywords}

- arthroplasty, replacement, knee

- quality of life

- osteoarthrosis

Objective To compare the function and quality of life of patients undergoing total knee arthroplasty (TKA) with fixed tibial platform and mobile tibial platform.

Methods We evaluated 240 patients with knee osteoarthritis, randomized into two groups - Group A consisted of 120 patients who underwent TKA with fixed tibial platform, and the B group, consisting of 120 patients who underwent mobile platform arthroplasty. Patients were accessed according to the function and quality of life by the Western Ontario and McMaster Universities Arthritis Index (WOMAC) and the Short Form Health Survey (SF-36), and pain scores by visual analog scale (VAS) of pain, preoperatively and at 6 months, 1 year, 2 years, 4 years and 8 years of surgery.

Results Regarding the various domains of the SF-36, we observed that the average behavior of functional capacity scores, physical aspects, pain and emotional aspects in the patient groups were statistically different during follow-up. The other domains of quality of life showed no mean differences. Regarding the pain assessed by VAS and WOMAC pain scores, we can see that it showed a mean change in follow-up in both patient groups. However, at 2 years of follow-up, they were statistically worse in group $A$, equaling group $B$ in the other moments.

Conclusion After 2 years of follow-up, we observed that pain scores and VAS were lower in the fixed platform group. However, these differences did not remain in the midterm, suggesting that the mobile tibial platform arthroplasty has a short-term advantage, and may help in the rehabilitation process.
\end{abstract}

\section{Introdução}

Nas últimas décadas, com o envelhecimento da população geral e as alterações do sistema musculoesquelético decorrentes desse processo, a osteoartrose tem se tornado um importante problema de saúde. ${ }^{1-3}$ Os sintomas desta doença degenerativa da cartilagem articular levam à incapacidade funcional e perda da qualidade de vida do idoso. ${ }^{4-9}$ Estes sintomas têm sido elementos de avaliação dos tratamentos, entre eles, a artroplastia total de joelho (ATJ). ${ }^{10,11}$

Os métodos para avaliar os resultados da ATJ são: as taxas de mortalidade, morbidade, complicações e durabilidade. Entretanto, com o crescimento rápido de melhorias nos procedimentos, essas taxas já não refletem o real benefício na qualidade de vida do indivíduo. ${ }^{12-14}$ Dessa forma, as avaliações com questionários genéricos ou específicos referentes ao tratamento têm fornecido informações valiosas. Destaca-se, entre eles, o Western Ontario and McMaster Universities Arthritis Index(WOMAC) para a ATJ, e o Short Form Health Survey(SF-36) para avaliar a qualidade de vida. ${ }^{10,15}$ Estes questionários têm mostrado os bons resultados da ATJ na melhora da função e qualidade de vida de pacientes idosos.

A ATJ pode ser dividida de acordo com o componente tibial em dois tipos: ATJ com plataforma fixa e com plataforma móvel. Segundo Wyldeet al, ${ }^{16}$ a ATJ padrão -com plataforma fixa- pode levar a uma carga excessiva na região posterior do componente tibial, aumentando o desgaste do polietileno, acarretando maior risco de falha e necessidade de revisão. Dessa forma, as ATJs com plataforma móvel, por permitirem maior mobilidade rotatória e melhor congruência do componente de polietileno, possuem a vantagem teórica de se autoalinhar, reduzindo a incidência de dor anterior no joelho, produzindo melhor função.

Visto isso, as vantagens teóricas da ATJ com plataforma móvel devem ser confirmadas clinicamente, já que, até agora, não há consenso em relação aos melhores resultados, e estudos anteriores foram considerados de baixa qualidade. ${ }^{17}$

O objetivo do presente estudo é comparar a função e qualidade de vida dos pacientes que foram submetidos à ATJ com plataformas fixa e móvel.

\section{Métodos}

Todos os procedimentos foram aprovados pelo comitê de ética em pesquisa da nossa universidade.

Este é um ensaio clínico randomizado, duplo-cego, realizado no período de janeiro de 2004 a janeiro de 2007. Os critérios de inclusão foram: 1-idade entre 55 e 70 anos, 2-sinais e sintomas clínicos compatíveis com osteoartrose do joelho, 3-sinais radiográficos de osteoartrose tricompartimental graus III, IV e V de Ahlback modificada por Keyes e Goodfellow, 4-Ausência de doenças associadas acometendo membros inferiores, 5-Ausência de distúrbio neurológico, 6-Ausência de lesões nervosas ou fraturas prévias nos membros inferiores. Os critérios de não-inclusão foram: 1-infecção, 2-deformidade de flexão $>10^{\circ}$, 3-desvios angulares em varo e valgo $>25^{\circ}$, 4-defeito focal tumoral, 5-condições físicas que eliminariam o suporte de implante adequado, 6-doença coexistente com risco à vida no ano seguinte ao procedimento. Pacientes que se disseram incapazes ou não deram certeza de retornar para acompanhamento foram excluídos do estudo. 
Após avaliação clínica e radiológica completa, os pacientes com indicação para ATJ que preenchiam os critérios foram convidados a participar do estudo. Os que confirmaram a participação assinaram o termo de consentimento livre e esclarecido. $\mathrm{O}$ método de randomização utilizado foi a permuta em blocos, com o objetivo de manter uma distribuição semelhante do número de pacientes em cada grupo estudado. Blocos de oito pacientes foram criados, com diferentes combinações. Envelopes lacrados, opacos e numerados de $1 \mathrm{a}$ 240 continham o grupo ao qual cada paciente pertencia: oprimeiro grupo (grupo A), submetido à ATJ com plataforma tibial fixa (DepuySynthes, Warsaw, IN, EUA), e o segundo grupo (grupo B), submetido à ATJ com plataforma tibial móvel (LCS, DepuySynthes, Warsaw, IN, EUA).

Todos os pacientes foram avaliados com questionários no pré-operatório e no pós-operatório com 6, 24, 48 e 96 meses com relação à função (WOMAC), qualidade de vida (SF-36) e percepção subjetiva da dor (Escala visual analógica de dor [EVA]).

\section{Tamanho da Amostra}

Para aceitar um risco alfa de 0.05 e um risco $\beta$ de $0.20,98$ pacientes foram necessários para cada grupo para detectar uma diferença $\geq 08$ pontos entre a média de pontuações pré e pós-operatórias para as dimensões de dor e função por meio do questionário de WOMAC, julgada uma diferença clinicamente importante. ${ }^{18}$ Um desvio padrão (DP) comum de 20 foi assumido. A amostra foi superestimada em $20 \%$ para permitir eventuais perdas, de tal forma que cada grupo deveria conter 120 pacientes.

\section{Técnica Cirúrgica}

Todas as próteses foram implantadas pelo mesmo cirurgião. Em todos os pacientes, realizou-se bloqueio anestésico do tipo raquimedular. Por 48horas, foi utilizada antibioticoterapia profilática com cefazolina sódica. Garrote pneumático foi usado rotineiramente. A via de acesso foi a anterior com artrotomia parapatelar medial. A patela foi evertida e substituída em todos os casos. Ambas as próteses tinham o componente femoral similar e todas eram estabilizadas posteriormente. Ambos os ligamentos cruzados foram extraídos. Realizava-se primeiro o corte ósseo tibial, horizontal, usando guia extramedular para tíbia e intramedular para o fêmur. Todos os componentes foram cimentados. Utilizou-se dreno de sucção por 24horas de rotina. Para a profilaxia tromboembólica, por 14 dias, os pacientes receberam heparina de baixo peso molecular.

\section{Reabilitação}

Preconizou-se uma rápida mobilização, em que, no primeiro dia de pós-operatório, foram realizados exercícios metabólicos de tornozelo e exercícios isométricos para quadríceps. No segundo dia de pós-operatório, depois de retirado o dreno de sucção, iniciou-se o treino de marcha com andador e descarga de peso em ambos os membros. 0 treino de marcha foi realizado de acordo com a tolerância de cada paciente (dor e condições clínicas). Todos os pacientes realizaram sessões de uma hora de movimento passivo continuo
(CPM), duas vezes ao dia (manhã e tarde), e o ângulo de movimento variava de acordo com a tolerância à dor de cada um. A alta hospitalar foi dada em média com 5 dias de pósoperatório e quando o paciente atingisse próximo dos $90^{\circ}$ de flexão do joelho e conseguisse deambular independentemente com muletas ou andador. As sessões de fisioterapias ambulatoriais começaram 1 semana após a alta hospitalar. 0 programa de reabilitação ambulatorial durou em média 2meses, sendo semelhantes para os 2grupos.

\section{Avaliação Clínica}

Realizou-se a avaliação da função por meio do WOMAC, sendo composto por três domínios: função, dor e rigidez. A soma dos pontos de cada domínio forma o resultado final, variando de 0 a 68. Para avaliar a qualidade de vida, foi usado o SF-36, variando de 0 a 100, apresentando 36 itens de resposta, envolvendo 8 conceitos: capacidade funcional, aspecto físico,dor, estado geral de saúde, vitalidade, aspectos sociais, aspectos emocionais e saúde mental. Aplicou-se também a EVA, variando de 0 a 10.

\section{Análises Estatísticas}

Foi verificada a existência de associação entre os tipos de prótese e as características com uso de testes qui-quadrado. ${ }^{19}$ As características quantitativas dos pacientes foram descritas segundo os tipos de prótese com uso de medidas resumo (média, DP, mediana e quartis, P25 e P75) e comparadas entre os grupos com uso de análise de testes $t$ de Student. ${ }^{19}$ Os escores das escalas avaliadas foram descritos segundo os tipos de prótese em cada momento de avaliação e comparados entre os tipos de prótese e momentos com uso de análises de equações de estimação generalizadas com distribuição marginal normal e função de ligação logarítmica, devido à assimetria de distribuição dos escores, supondo correlação autorregressiva de primeira ordem entre os momentos de avaliação. ${ }^{20}$ As análises foram seguidas de comparações múltiplas de Bonferroni ${ }^{21}$ para comparar os grupos e momentos, quando significativas as diferenças nos escores. As análises foram realizadas com os dados avaliados nos pacientes, mesmo considerando perdas durante o seguimento. Os resultados foram ilustrados com gráficos de perfis médios, com os respectivos errospadrão, tendo sido os testes realizados com nível de significância de $5 \%$.

\section{Resultados}

Os pacientes foram recrutados consecutivamente de novembro de 2011 até dezembro de 2012. No total, 1.268 pacientes foram avaliados e 1.028 foram excluídos, resultando em uma amostra final de 240 pacientes. Os pacientes foram randomizados em 2grupos: 120 na ATJ com plataforma fixa e 120 na ATJ com plataforma móvel. Do grupo com plataforma fixa, cinco pacientes evoluíram para óbito, e seis não aderiram. Do grupo com plataforma móvel, seis evoluíram para óbito, quatro não aderiram, um teve acidente vascular cerebral (AVC)e um teve ruptura do ligamento patelar. Todos os óbitos ocorreram após $>2$ anos de seguimento (-Figura 1). 


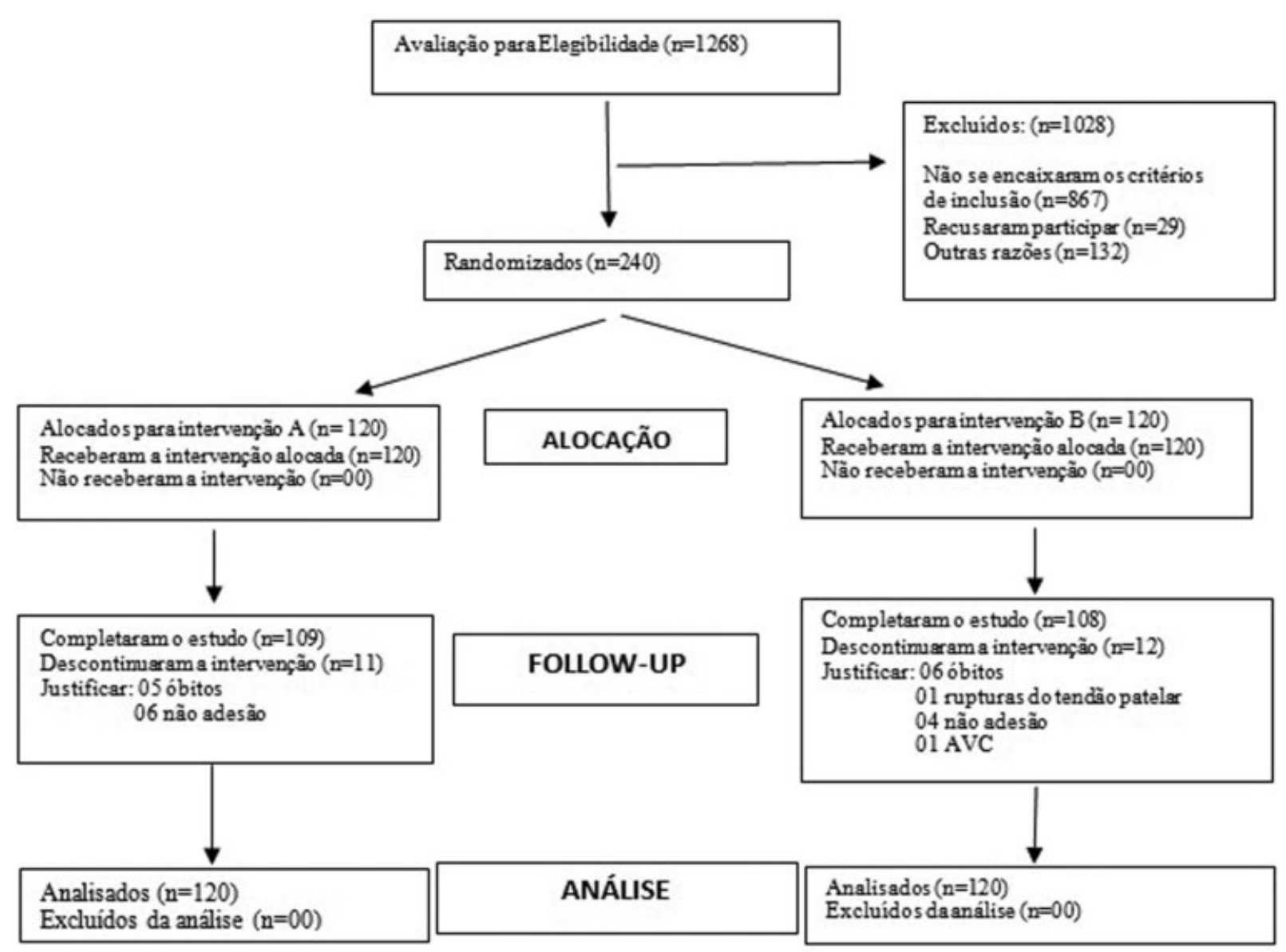

Fig. 1 Fluxograma de fases.

Dos 240 pacientes randomizados, 6do grupo com plataforma fixa e 5 do grupo com plataforma móvel apresentaram complicações. No grupo com plataforma fixa, tivemos três casos de infecção, dois com embolia e um com trombose venosa profunda. No grupo com plataforma móvel, tivemos dois casos de infecção, um de trombose venosa profunda, um de AVCe um com ruptura do ligamento patelar.

As idades dos indivíduos da amostra foram compreendidas entre 59 e 70 anos, com média de 65,7 anos ( $\mathrm{DP}=3,7$ ). Eram do gênero feminino 81\%, com índice de massa corporal (IMC) de 30 ( $\mathrm{DP}=4,7$ ). As características pessoais avaliadas não apresentaram associação ou diferenças estatisticamente significantes;portanto, os grupos foram homogêneos, como mostra a - Tabela 1.

No que diz respeito aos diversos domínios do questionário de qualidade de vida do SF-36, não foi mostrada diferença entre os grupos na qualidade de vida ao final do seguimento. Apenas em alguns domínios observa-se diferença entre os grupos, em determinados momentos. Um exemplo está no escore de dor em 1e 2anos de seguimento, mas que parece igualar nos demais momentos.

A - Tabela 2 mostra que o comportamento médio dos escores de capacidade funcional, dor e aspectos emocionais foram estatisticamente diferentes ao longo do seguimento nos grupos de pacientes, conforme os valores realçados na tabela. Os demais domínios de qualidade de vida apresenta- ram diferenças médias apenas ao longo do seguimento, nos diferentes momentos de avaliação, porém sem diferença entre os grupos.

$\mathrm{Na}$ - Tabela 3, a EVA de dor e os escores de dor do WOMAC apresentaram, em média, comportamento estatisticamente diferente entre os grupos ao longo do seguimento $(p<0,001)$. No escore de função e rigidez do WOMAC, houve diferença média estatisticamente significante apenas ao longo do seguimento, nos diferentes momentos de avaliação, sem diferença entre os grupos $(p<0,001)$.

\section{Discussão}

O presente estudo prospectivo, randomizado e controlado constatou que, com 8anos de pós-operatório, não houve diferenças significativas no desfecho clínico dor dos questionários de qualidade de vida SF-36 e WOMAC, bem como nas pontuações da EVA após cirurgias de próteses de joelho com plataforma tibial fixa em relação aos implantes de plataforma móvel. Recentes estudos randomizados prospectivos ${ }^{16,22,23}$ também não conseguiram constatar diferença na evolução clínica, análise radiológica ou sobrevivência entre as próteses fixa e móvel. Esses mesmos autores compararam os resultados clínicos dos dois tipos de implante no mesmo paciente e não encontraram diferenças nos escores de dor e amplitude de movimento articular (ADM) com 5anos de 
Função e Qualidade de Vida de Pacientes Submetidos à Artroplastia Total do Joelho Nacca et al. 57

Tabela 1 Descrição das características pessoais dos pacientes segundo tipos de prótese e resultados dos testes estatísticos

\begin{tabular}{|c|c|c|c|c|}
\hline \multirow[t]{2}{*}{ Variável } & \multicolumn{2}{|l|}{ Tipo de prótese } & \multirow[t]{2}{*}{ Total $(N=240)$} & \multirow[t]{2}{*}{ valor-p } \\
\hline & Fixa $(N=120)$ & Móvel $(N=120)$ & & \\
\hline \multicolumn{5}{|l|}{ Gênero, n (\%) } \\
\hline Feminino & $96(80)$ & $100(83,3)$ & $196(81,7)$ & \multirow[t]{2}{*}{$0,505^{*}$} \\
\hline Masculino & $24(20)$ & $20(16,7)$ & $44(18,3)$ & \\
\hline \multicolumn{5}{|l|}{ Ladooperado, n (\%) } \\
\hline Direito & $64(53,3)$ & $57(47,5)$ & $121(50,4)$ & \multirow[t]{2}{*}{$0,366^{*}$} \\
\hline Esquerdo & $56(46,7)$ & $63(52,5)$ & $119(49,6)$ & \\
\hline \multicolumn{5}{|l|}{ Idade (anos) } \\
\hline média (DP) & $65,9(3,9)$ & $65,4(3,4)$ & $65,7(3,7)$ & \multirow[t]{2}{*}{0,369} \\
\hline mediana (P25; P75) & $66(63 ; 70)$ & $65(63 ; 68)$ & $65(63 ; 69)$ & \\
\hline \multicolumn{5}{|l|}{ Peso $(\mathrm{Kg})$} \\
\hline média (DP) & $78,4(12,1)$ & $80,3(13,5)$ & $79,4(12,8)$ & \multirow[t]{2}{*}{0,276} \\
\hline mediana (P25; P75) & $78,5(73 ; 85)$ & $78,5(71 ; 87)$ & $78,5(72 ; 86)$ & \\
\hline \multicolumn{5}{|l|}{ Altura (m) } \\
\hline média (DP) & $1,62(0,06)$ & $1,62(0,14)$ & $1,62(0,10)$ & \multirow[t]{2}{*}{0,320} \\
\hline mediana (P25; P75) & $1,63(1,6 ; 1,66)$ & $1,63(1,57 ; 1,67)$ & $1,63(1,59 ; 1,67)$ & \\
\hline \multicolumn{5}{|l|}{$\mathrm{IMC}(\mathrm{Kg} / \mathrm{m} 2)$} \\
\hline média (DP) & $29,7(4,2)$ & $30,2(5,1)$ & $30(4,7)$ & \multirow[t]{2}{*}{0,426} \\
\hline mediana (P25; P75) & $29,5(27,2 ; 32,3)$ & $30,1(27,2 ; 33,1)$ & $29,7(27,2 ; 32,9)$ & \\
\hline
\end{tabular}

Abreviações: DP, desvio padrão; IMC, índice de massa corporal.

Teste t de Student; * Teste qui-quadrado.

Tabela 2 Descrição dos escores de qualidade de vida segundo tipos de prótese e momentos de avaliação e resultados estatísticos

\begin{tabular}{|c|c|c|c|c|c|c|c|c|c|c|}
\hline \multirow[t]{3}{*}{ Variável } & \multirow[t]{3}{*}{ Momento } & \multicolumn{6}{|c|}{ Tipo de prótese } & \multirow{3}{*}{$\begin{array}{l}\text { valor-p Tipo } \\
\text { de prótese }\end{array}$} & \multirow{3}{*}{$\begin{array}{l}\text { valor-p } \\
\text { Momento }\end{array}$} & \multirow{3}{*}{$\begin{array}{l}\text { valor-p } \\
\text { Interação }\end{array}$} \\
\hline & & \multicolumn{3}{|l|}{ Fixa } & \multicolumn{3}{|l|}{ Móvel } & & & \\
\hline & & Média & $\mathrm{DP}$ & $\mathrm{N}$ & Média & DP & $\mathrm{N}$ & & & \\
\hline \multirow[t]{6}{*}{ Capacidade Funcional } & Pré & 20,22 & 18,10 & 120 & 17,17 & 14,79 & 120 & \multirow[t]{6}{*}{0,219} & \multirow[t]{6}{*}{$<0,001$} & \multirow[t]{6}{*}{0,019} \\
\hline & 6 meses & 57,54 & 19,47 & 120 & 62,79 & 19,39 & 120 & & & \\
\hline & 1 ano & 65,46 & 15,38 & 120 & 74,42 & 17,32 & 120 & & & \\
\hline & 2 anos & 68,37 & 16,76 & 120 & 73,88 & 16,71 & 120 & & & \\
\hline & 4 anos & 61,63 & 15,46 & 120 & 63,88 & 15,14 & 120 & & & \\
\hline & 8 anos & 51,46 & 16,72 & 120 & 55,46 & 16,09 & 120 & & & \\
\hline \multirow[t]{6}{*}{ Aspectos Físicos } & Pré & 13,48 & 26,58 & 120 & 17,23 & 25,76 & 120 & \multirow[t]{6}{*}{0,910} & \multirow[t]{6}{*}{$<0,001$} & \multirow[t]{6}{*}{0,536} \\
\hline & 6 meses & 71,68 & 28,24 & 120 & 67,86 & 37,68 & 120 & & & \\
\hline & 1 ano & 77,08 & 24,38 & 120 & 73,96 & 31,49 & 120 & & & \\
\hline & 2 anos & 88,27 & 65,30 & 120 & 82,75 & 29,13 & 120 & & & \\
\hline & 4 anos & 84,77 & 23,00 & 120 & 75,43 & 35,65 & 120 & & & \\
\hline & 8 anos & 78,93 & 25,52 & 120 & 78,34 & 28,06 & 120 & & & \\
\hline \multirow[t]{5}{*}{ Dor } & Pré & 41,89 & 26,12 & 120 & 35,34 & 22,69 & 120 & \multirow[t]{5}{*}{0,053} & \multirow[t]{5}{*}{$<0,001$} & \multirow[t]{5}{*}{0,004} \\
\hline & 6 meses & 80,19 & 26,03 & 120 & 79,39 & 23,79 & 120 & & & \\
\hline & 1 ano & 75,41 & 30,97 & 120 & 86,20 & 18,29 & 120 & & & \\
\hline & 2 anos & 69,63 & 34,84 & 120 & 92,49 & 86,12 & 120 & & & \\
\hline & 4 anos & 76,72 & 28,09 & 120 & 81,05 & 19,50 & 120 & & & \\
\hline
\end{tabular}


Tabela 2 (Continua)

\begin{tabular}{|c|c|c|c|c|c|c|c|c|c|c|}
\hline \multirow[t]{3}{*}{ Variável } & \multirow[t]{3}{*}{ Momento } & \multicolumn{6}{|c|}{ Tipo de prótese } & \multirow{3}{*}{$\begin{array}{l}\text { valor-p Tipo } \\
\text { de prótese }\end{array}$} & \multirow{3}{*}{$\begin{array}{l}\text { valor-p } \\
\text { Momento }\end{array}$} & \multirow{3}{*}{$\begin{array}{l}\text { valor-p } \\
\text { Interação }\end{array}$} \\
\hline & & \multicolumn{3}{|l|}{ Fixa } & \multicolumn{3}{|l|}{ Móvel } & & & \\
\hline & & Média & DP & $\mathrm{N}$ & Média & DP & $\mathrm{N}$ & & & \\
\hline & 8 anos & 74,07 & 27,78 & 120 & 85,28 & 62,49 & 120 & & & \\
\hline \multirow[t]{6}{*}{ Estado Geral de Saúde } & Pré & 69,58 & 18,19 & 120 & 71,60 & 16,32 & 120 & \multirow[t]{6}{*}{0,512} & \multirow[t]{6}{*}{$<0,001$} & \multirow[t]{6}{*}{0,167} \\
\hline & 6 meses & 77,96 & 13,10 & 120 & 80,53 & 12,77 & 120 & & & \\
\hline & 1 ano & 78,10 & 12,54 & 120 & 76,38 & 15,23 & 120 & & & \\
\hline & 2 anos & 76,18 & 12,89 & 120 & 72,29 & 18,29 & 120 & & & \\
\hline & 4 anos & 77,21 & 14,26 & 120 & 75,18 & 15,45 & 120 & & & \\
\hline & 8 anos & 74,88 & 14,10 & 120 & 73,51 & 14,69 & 120 & & & \\
\hline \multirow[t]{6}{*}{ Vitalidade } & Pré & 68,03 & 18,79 & 120 & 68,45 & 19,16 & 120 & \multirow[t]{6}{*}{0,366} & \multirow[t]{6}{*}{$<0,001$} & \multirow[t]{6}{*}{0,779} \\
\hline & 6 meses & 78,64 & 14,60 & 120 & 78,14 & 15,10 & 120 & & & \\
\hline & 1 ano & 79,49 & 13,16 & 120 & 77,23 & 16,89 & 120 & & & \\
\hline & 2 anos & 78,36 & 14,18 & 120 & 75,43 & 17,09 & 120 & & & \\
\hline & 4 anos & 78,85 & 14,14 & 120 & 77,98 & 15,59 & 120 & & & \\
\hline & 8 anos & 79,56 & 16,12 & 120 & 77,98 & 15,93 & 120 & & & \\
\hline \multirow[t]{6}{*}{ Aspectos Sociais } & Pré & 45,90 & 26,75 & 120 & 50,80 & 27,66 & 120 & \multirow[t]{6}{*}{0,062} & \multirow[t]{6}{*}{$<0,001$} & \multirow[t]{6}{*}{0,080} \\
\hline & 6 meses & 81,65 & 23,33 & 120 & 86,17 & 20,40 & 120 & & & \\
\hline & 1 ano & 84,28 & 23,08 & 120 & 87,68 & 20,49 & 120 & & & \\
\hline & 2 anos & 85,06 & 21,43 & 120 & 84,22 & 23,49 & 120 & & & \\
\hline & 4 anos & 83,60 & 22,30 & 120 & 84,38 & 24,01 & 120 & & & \\
\hline & 8 anos & 78,95 & 25,64 & 120 & 87,13 & 20,68 & 120 & & & \\
\hline \multirow[t]{6}{*}{ Aspectos Emocionais } & Pré & 52,68 & 45,09 & 120 & 40,26 & 45,11 & 120 & \multirow[t]{6}{*}{0,771} & \multirow[t]{6}{*}{$<0,001$} & \multirow[t]{6}{*}{0,036} \\
\hline & 6 meses & 73,54 & 36,84 & 120 & 81,40 & 35,59 & 120 & & & \\
\hline & 1 ano & 79,74 & 34,68 & 120 & 86,65 & 29,79 & 120 & & & \\
\hline & 2 anos & 92,98 & 109,76 & 120 & 86,61 & 30,13 & 120 & & & \\
\hline & 4 anos & 86,88 & 90,35 & 120 & 86,08 & 29,47 & 120 & & & \\
\hline & 8 anos & 81,36 & 30,33 & 120 & 87,21 & 27,42 & 120 & & & \\
\hline \multirow[t]{6}{*}{ Saúde Mental } & Pré & 71,04 & 20,07 & 120 & 73,81 & 18,15 & 120 & \multirow[t]{6}{*}{0,317} & \multirow[t]{6}{*}{$<0,001$} & 0,484 \\
\hline & 6 meses & 77,27 & 17,25 & 120 & 76,85 & 16,31 & 120 & & & \\
\hline & 1 ano & 76,87 & 17,04 & 120 & 77,20 & 13,12 & 120 & & & \\
\hline & 2 anos & 75,05 & 19,15 & 120 & 75,99 & 14,34 & 120 & & & \\
\hline & 4 anos & 75,09 & 19,65 & 120 & 78,43 & 14,58 & 120 & & & \\
\hline & 8 anos & 73,58 & 19,55 & 120 & 75,90 & 16,25 & 120 & & & \\
\hline
\end{tabular}

Abreviação: DP, desvio padrão.

seguimento. Aglietti et al, ${ }^{24}$ em seu estudo sobre pacientes submetidos a artroplastia unilateral de joelho que compara os dois tipos de próteses, também não observaram diferenças significativas com 3anos de seguimento nos escores de dor, embora maior flexão tenha sido apontada em joelhos com plataforma tibial fixa. É possível que a falta de diferença nos resultados clínicos após 8 anos de seguimento encontrados no presente estudo entre implantes com plataforma fixa e plataforma móvel deveu-se às características dos participantes, principalmente no que tange a faixa etária.

Além disso, o instrumento genérico para avaliação da qualidade de vida do SF-36 de pacientes nesta faixa etária contribui para confirmar estes resultados, mas nos parece insuficiente quando usado de maneira isolada para estabelecer conclusões do ponto de vista clínico. Quando analisada a dor, alguns pacientes confundem-se, pois, a questão relaciona-se com "dor no corpo." Tanto as questões de dor, como aspectos emocionais, disposição, vitalidade, foram frequentemente respondidas positivamente, mas praticamente em todos os casos houve dificuldade para relacionarem a resposta diretamente ao joelho, por serem questões de abrangência maior.

A média de idade dos participantes do presente estudo foi de $65,7(\mathrm{DP}=3,7)$ anos, e a maioria não realizava atividades 
Função e Qualidade de Vida de Pacientes Submetidos à Artroplastia Total do Joelho Nacca et al. 59

Tabela 3 Descrição da escala visual analógicade dor e dos escores de funcionalidade segundo os tipos de prótese e momentos de avaliação e resultados estatísticos

\begin{tabular}{|c|c|c|c|c|c|c|c|c|c|c|}
\hline \multirow[t]{3}{*}{ Variável } & \multirow[t]{3}{*}{ Momento } & \multicolumn{6}{|c|}{ Tipo de prótese } & \multirow{3}{*}{$\begin{array}{l}\text { valor-pTipo } \\
\text { de prótese }\end{array}$} & \multirow{3}{*}{$\begin{array}{l}\text { valor-p } \\
\text { Momento }\end{array}$} & \multirow{3}{*}{$\begin{array}{l}\text { valor-p } \\
\text { Interação }\end{array}$} \\
\hline & & \multicolumn{3}{|l|}{ Fixa } & \multicolumn{3}{|l|}{ Móvel } & & & \\
\hline & & Média & DP & $\mathrm{N}$ & Média & DP & $\mathrm{N}$ & & & \\
\hline \multirow[t]{6}{*}{ EVA de Dor } & Pré & 84,69 & 17,04 & 120 & 85,40 & 17,49 & 120 & \multirow[t]{6}{*}{0,016} & \multirow[t]{6}{*}{$<0,001$} & \multirow[t]{6}{*}{$<0,001$} \\
\hline & 6 meses & 26,43 & 22,13 & 120 & 24,70 & 22,43 & 120 & & & \\
\hline & 1 ano & 25,63 & 15,08 & 120 & 20,53 & 20,85 & 120 & & & \\
\hline & 2 anos & 28,83 & 19,40 & 120 & 16,57 & 18,97 & 120 & & & \\
\hline & 4 anos & 14,06 & 17,39 & 120 & 13,04 & 18,07 & 120 & & & \\
\hline & 8 anos & 13,78 & 16,37 & 120 & 10,56 & 16,59 & 120 & & & \\
\hline \multirow[t]{6}{*}{ WOMAC de Dor } & Pré & 13,60 & 3,86 & 120 & 14,04 & 3,42 & 120 & \multirow[t]{6}{*}{0,032} & \multirow[t]{6}{*}{$<0,001$} & \multirow[t]{6}{*}{$<0,001$} \\
\hline & 6 meses & 3,46 & 3,53 & 120 & 3,42 & 3,82 & 120 & & & \\
\hline & 1 ano & 3,86 & 3,30 & 120 & 2,67 & 3,91 & 120 & & & \\
\hline & 2 anos & 5,11 & 3,97 & 120 & 2,86 & 4,01 & 120 & & & \\
\hline & 4 anos & 2,91 & 3,06 & 120 & 2,24 & 3,92 & 120 & & & \\
\hline & 8 anos & 2,31 & 3,21 & 120 & 1,77 & 3,72 & 120 & & & \\
\hline \multirow[t]{6}{*}{ WOMAC função } & Pré & 43,64 & 13,91 & 120 & 45,36 & 12,60 & 120 & \multirow[t]{6}{*}{0,037} & \multirow[t]{6}{*}{$<0,001$} & \multirow[t]{6}{*}{0,001} \\
\hline & 6 meses & 14,11 & 11,78 & 120 & 10,94 & 9,45 & 120 & & & \\
\hline & 1 ano & 9,62 & 9,81 & 120 & 8,53 & 7,79 & 120 & & & \\
\hline & 2 anos & 8,83 & 9,81 & 120 & 7,11 & 7,39 & 120 & & & \\
\hline & 4 anos & 13,12 & 9,81 & 120 & 10,13 & 7,96 & 120 & & & \\
\hline & 8 anos & 21,37 & 10,90 & 120 & 18,31 & 8,47 & 120 & & & \\
\hline \multirow[t]{6}{*}{ WOMAC rigidez } & Pré & 4,40 & 2,43 & 120 & 5,01 & 2,34 & 120 & \multirow[t]{6}{*}{0,198} & \multirow[t]{6}{*}{$<0,001$} & \multirow[t]{6}{*}{0,203} \\
\hline & 6 meses & 1,38 & 1,66 & 120 & 1,31 & 1,40 & 120 & & & \\
\hline & 1 ano & 0,98 & 1,49 & 120 & 0,99 & 1,25 & 120 & & & \\
\hline & 2 anos & 0,88 & 1,49 & 120 & 0,58 & 1,00 & 120 & & & \\
\hline & 4 anos & 0,94 & 1,43 & 120 & 0,63 & 1,02 & 120 & & & \\
\hline & 8 anos & 0,93 & 1,49 & 120 & 0,68 & 1,04 & 120 & & & \\
\hline
\end{tabular}

Abreviação: DP, desvio padrão; EVA, escala visual analógica; WOMAC, Western Ontario andMcMasterUniversitiesArthritis Index.

físicas de recreação ou esportivas que demandassem maior grau de movimento articular. Segundo Wylde et al, ${ }^{16}$ a prótese de suporte tibial móvel foi concebida para proporcionar uma maior amplitude de movimento articular e permitir a participação em atividades que exijam uma maior mobilidade do joelho em todos os planos. Portanto, pode-se argumentar que o implante de apoio tibial móvel do joelho não atingiu seu pleno potencial nesse grupo de pacientes por tratar-se de estudo de uma população mais velha. Um ensaio clínico randomizado envolvendo pacientes mais jovens e mais ativos poderia revelar alguma vantagem funcional de um desenho sobre o outro.

Um achado importante deste estudo a destacar é o fato de que, em curto seguimento de tempo -2anos após a cirurgia, os escores de dor da EVA e WOMAC foram significativamente piores no grupo com plataforma tibial fixa $(p<0,05$ e $p<0,001$, respectivamente). Nesse momento, os piores escores de dor influenciaram negativamente na qualidade de vida em pacientes submetidos a ATJ com plataforma tibial fixa.
Ao mesmo tempo, nota-se paradoxalmente que, exatamente nesse período com 2anos de seguimento, os grupos tiveram os melhores escores da capacidade funcional, tanto nas avaliações do questionário de qualidade de vida SF-36 como nas avaliações funcionais do questionário de WOMAC, sem diferenças estatísticas significantes entre os grupos fixa e móvel.

Apesar de a ATJ já ter demonstrado ser um procedimento bem-sucedido para o tratamento de pacientes com osteoartrose, uma percentagem significativa ainda pode experimentar dor após a cirurgia. ${ }^{25}$ Embora os resultados dos ensaios clínicos randomizados ainda não serem conclusivos para determinar se o tipo de implante pode influenciar na dor no joelho pós-operatória, os dados obtidos no presente estudo sugerem que, em 2anos de seguimento, o domínio dor do SF-36 influenciou menos na qualidade de vida no grupo de submetidos a ATJ com plataforma tibial móvel quando comparado ao grupo submetido à prótese total com plataforma fixa. 
Aglietti et $\mathrm{al}^{24}$ sugeriam que as vantagens de um projeto com apoio tibial móvel podem diminuir ao longo do tempo. Também se observa isso no presente estudo, em que, após 2anos de cirurgia, parece que os escores de dor se alinham novamente, não se registrando diferenças estatísticas significantes entre os grupos com 4 e 8 anos quanto aos níveis de dor. No entanto, como dor anterior do joelho é relevante para os pacientes mesmo num curto prazo, não se acredita que isso configure uma limitação para uso das ATJ com plataforma móvel.

Destaca-se como ponto forte deste estudo o fato de que todas as cirurgias foram realizadas pelo mesmo cirurgião, com experiência nos dois tipos de ATJ, minimizando os fatores de viés. Além disso, o seguimento é de médio a longo prazo, com um tamanho de amostra maior do que da maioria dos estudos anteriores. A fim de se reduzir o viés da aplicação, os questionários foram preenchidos pelos próprios pacientes, com ajuda do avaliador. As avaliações em todos os momentos foram realizadas por um fisioterapeuta que não sabia para qual grupo os pacientes tinham sido randomizados.

Como limitação da presente análise, pode-se citar a não divisão dos pacientes de acordo com a ADM prévia ao procedimento cirúrgico e final. Também não se realizou a análise radiológica no sentido de aferir vantagens de um implante sobre o outro com relação ao aspecto soltura, o que não era um objetivo da presentepesquisa.

Ao idealizar o presente estudo, o foco principal era averiguar se existiam diferenças funcionais e na qualidade de vida em um grupo de idosos com osteoartrose de joelho submetidos aos dois tipos de ATJ. No entanto, durante sua realização, algumas indagações surgiram e permanecem sem resposta, necessitando ser investigadas.

\section{Conclusão}

Os dados do presente estudo demonstram que com 2anos de pós-operatório os escores de dor nos questionários SF-36, EVA e WOMAC foram piores no grupo ATJ plataforma fixa. Porém, os indivíduos submetidos à ATJ com plataforma tibial fixa não apresentaram diferenças funcionais e na qualidade de vida em comparação àqueles submetidos à artroplastia com plataforma tibial móvel, com seguimento de médio prazo.

\section{Conflito de Interesses}

Os autores declaram não haver conflito de interesses.

\section{Referências}

1 Lanza IR, Towse TF, Caldwell GE, Wigmore DM, Kent-Braun JA. Effects of age on human muscle torque, velocity, and power in two muscle groups. J Appl Physiol (1985) 2003;95(06):2361-2369

2 Narici MV, Maganaris C, Reeves N. Myotendinous alterations and effects of resistive loading in old age. Scand J Med Sci Sports 2005; 15(06):392-401

3 Herzog W, Longino D. The role of muscles in joint degeneration and osteoarthritis. J Biomech 2007;40(Suppl 1):S54-S63

4 Laborde JM, Powers MJ. Life satisfaction, health control orientation, and illness-related factors in persons with osteoarthritis. Res Nurs Health 1985;8(02):183-190
5 Downe-Wamboldt B. Coping and life satisfaction in elderly women with osteoarthritis. J Adv Nurs 1991;16(11):1328-1335

6 Dickstein R, Heffes Y, Shabtai EI, Markowitz E. Total knee arthroplasty in the elderly: patients' self-appraisal 6 and 12 months postoperatively. Gerontology 1998;44(04):204-210

7 Heck DA, Robinson RL, Partridge CM, Lubitz RM, Freund DA. Patient outcomes after knee replacement. Clin Orthop Relat Res 1998;(356):93-110

8 Bachmeier CJ, March LM, Cross MJ, et al. Arthritis Cost and Outcome Project Group. A comparison of outcomes in osteoarthritis patients undergoing total hip and knee replacement surgery. Osteoarthritis Cartilage 2001;9(02):137-146

9 Hartley RC, Barton-Hanson NG, Finley R, Parkinson RW. Early patient outcomes after primary and revision total knee arthroplasty. A prospective study. J Bone Joint Surg Br 2002;84(07):994-999

10 Mäntyselkä P, Kumpusalo E, Ahonen R, Takala J. Patients' versus general practitioners' assessments of pain intensity in primary care patients with non-cancer pain. BrJ Gen Pract 2001;51(473):995-997

11 Janse AJ, Gemke RJ, Uiterwaal CS, van der Tweel I, Kimpen JL, Sinnema G. Quality of life: patients and doctors don't always agree: a meta-analysis. J Clin Epidemiol 2004;57(07):653-661

12 O'Boyle CA. Assessment of quality of life in surgery. Br J Surg 1992; 79(05):395-398

13 Givon U, Ginsberg GM, Horoszowski H, Shemer J. Cost-utility analysis of total hip arthroplasties. Technology assessment of surgical procedures by mailed questionnaires. Int J Technol Assess Health Care 1998;14(04):735-742

14 Ethgen $O$, Bruyère $O$, Richy F, Dardennes C, Reginster JY. Healthrelated quality of life in total hip and total knee arthroplasty. A qualitative and systematic review of the literature. J Bone Joint Surg Am 2004;86(05):963-974

15 Ware J Jr, Kosinski M, Keller SDA. A 12-Item Short-Form Health Survey: construction of scales and preliminary tests of reliability and validity. Med Care 1996;34(03):220-233

16 Wylde V, Learmonth I, Potter A, Bettinson K, Lingard E. Patientreported outcomes after fixed- versus mobile-bearing total knee replacement: a multi-centre randomised controlled trial using the Kinemax total knee replacement. J Bone Joint Surg Br 2008;90 (09):1172-1179

17 Jacobs W, Anderson P, Limbeek J, Wymenga A. Mobile bearing vs fixed bearing prostheses for total knee arthroplasty for post-operative functional status in patients with osteoarthritis and rheumatoid arthritis. Cochrane Database Syst Rev 2004;(02):CD003130

18 Núñez M, Núñez E, del Val JL, et al. Health-related quality of life in patients with osteoarthritis after total knee replacement: factors influencing outcomes at 36 months of follow-up. Osteoarthritis Cartilage 2007;15(09):1001-1007

19 Kirkwood BR, Sterne JA. Essential medical statistics. 2nd ed. Massachusetts, USA: Blackwell; 2006

20 McCullagh P, Nelder JA. Generalized linear models. 2nd ed. New York, USA: Chapman and Hall; 1989

21 Neter J, Kutner MH, Nachtsheim CJ, Wasserman W. Applied Linear Statistical Models. 4th ed. Ilinois: Richard D. Irwing; 1996

22 Bhan S, Malhotra R, Kiran EK, Shukla S, Bijjawara M. A comparison of fixed-bearing and mobile-bearing total knee arthroplasty at a minimum follow-up of 4.5 years. J Bone Joint Surg Am 2005;87 (10):2290-2296

23 Matsuda S, Mizu-uchi H, Fukagawa S, et al. Mobile-bearing prosthesis did not improve mid-term clinical results of total knee arthroplasty. Knee Surg Sports Traumatol Arthrosc 2010;18(10):1311-1316

24 Aglietti P, Baldini A, Buzzi R, Lup D, De Luca L. Comparison of mobilebearing and fixed-bearing total knee arthroplasty: a prospective randomized study. J Arthroplasty 2005;20(02):145-153

25 Hofmann S, Seitlinger G, Djahani O, Pietsch M. The painful knee after TKA: a diagnostic algorithm for failure analysis. Knee Surg Sports Traumatol Arthrosc 2011;19(09):1442-1452 